\title{
LOCALLY COMPACT SIMPLE RINGS HAVING MINIMAL LEFT IDEALS
}

\author{
BY \\ SETH WARNER( $\left.{ }^{1}\right)$
}

Let $A$ be an indiscrete locally compact primitive ring having minimal left ideals. Algebraically, $A$ may be regarded as a dense ring of linear operators containing nonzero linear operators of finite rank on a vector space $E$ over a division $\operatorname{ring} K$, and furthermore if $A$ is simple, then $A$ contains only linear operators of finite rank. The topology of $A$ induces topologies on $E$ and $K$ in a natural way, so that $K$ becomes a locally compact division ring and $E$ a locally compact vector space over $K$. Theorems about locally compact division rings and locally compact vector spaces imply that if $K$ is indiscrete, then $E$ is necessarily finite dimensional over $K$, and thus $A$ is the ring of all linear operators on a finite-dimensional vector space over a locally compact division ring. Kaplansky proved that if $A$ has characteristic zero, then $K$ is indeed indiscrete, but he showed by an example that $E$ could be infinite dimensional. Kaplansky remarked [9, p. 459], however, that if $A$ is simple, it seemed unlikely that $E$ could be infinite dimensional, since otherwise "completeness appears to require the presence of linear transformations with infinitedimensional range." Our principal result in $\S 1$ is the verification of this conjecture; however, our argument is based not on the fact that a locally compact ring is complete, but rather on the fact that a locally compact space is a Baire space and on the symmetry between minimal left and right ideals in a simple ring. In $\$ 2$ we shall present certain results about locally compact vector spaces over discrete division rings; these enable us to prove that a locally compact, indiscrete, central primitive algebra having minimal left ideals is finite dimensional provided its scalar field either is indiscrete, has characteristic zero, or is uncountably infinite. However, there is a very natural example of a locally compact, indiscrete, infinitedimensional central primitive algebra having minimal left ideals over a discrete countably infinite field of prime characteristic.

1. Locally compact simple rings having minimal left ideals. We begin with a summary of the structure theory of a primitive ring $A$ having minimal left ideals. Let $e$ be a minimal idempotent of $A$, that is, an idempotent such that $A e$ is a minimal left ideal, or equivalently, such that $e A$ is a minimal right ideal. Then $e A e$ is a division ring, the additive group $A e$ becomes a right $e A e$-vector space by defining scalar multiplication to be the restriction of multiplication on $A$ to

Received by the editors September 16, 1965.

(1) Research supported by the National Science Foundation under grant GP 1913. 
$A e \times e A e$, and similarly $e A$ becomes a left $e A e$-vector space by defining scalar multiplication to be the restriction of multiplication on $A$ to $e A e \times e A$. For each $a \in A$, let $a_{L}$ be the linear operator on the right $e A e$-vector space $A e$ defined by $a_{L}(x)=a x$, and let $a_{R}$ be the linear operator on the left $e A e$-vector space $e A$ defined by $a_{R}(x)=x a$. Then $a \rightarrow a_{L}$ is an isomorphism from $A$ onto a dense ring $A_{L}$ of linear operators on $A e, a \rightarrow a_{R}$ is an anti-isomorphism from $A$ onto a dense ring $A_{R}$ of linear operators on $e A$, and both $A_{L}$ and $A_{R}$ contain nonzero linear operators of finite rank [7, Chapters 2 and 4].

Let us assume further that $A$ is a locally compact ring. If $\mathscr{V}$ is a fundamental system of neighborhoods of zero in $A$, then $\{V e: V \in \mathscr{V}\}$ is a fundamental system of neighborhoods of zero in $A e$. Indeed, if $V \in \mathscr{V}$, then $V e$ is a neighborhood of zero in $A e$ since $V \cap A e \subseteq V e$; conversely, if $U$ is a neighborhood of zero in $A$, then there is a neighborhood $W$ of zero in $A$ such that $W e \subseteq U \cap A e$ as $x \rightarrow x e$ is continuous. Similarly, $\{e V: V \in \mathscr{V}\}$ and $\{e V e: V \in \mathscr{V}\}$ are fundamental systems of neighborhoods of zero in $e A$ and $e A e$ respectively. If $V \in \mathscr{V}$ is compact, then $V e, e V$, and $e V e$ are also compact since they are continuous images of $V$; hence $A e, e A$, and $e A e$ are all locally compact. A theorem of Otobe [10, Theorem 3], generalized in several ways by Kaplansky [9, Theorems 7-9], implies that $x \rightarrow x^{-1}$ is continuous on the set of nonzero elements of $e A e$, and hence $e A e$ is a locally compact division ring; this also follows from a theorem of R. Ellis [4, p. 78, Exercise 25] applied to the multiplicative group of nonzero elements of $e A e$. Since the scalar multiplications of the vector spaces $A e$ and $e A$ are simply restrictions of multiplication on $A$, they are locally compact topological vector spaces over $e A e$. The topology of $e A e$ is defined by an absolute value [8, Theorem 8], and hence if $e A e$ is indiscrete, then $A e$ and $e A$ are necessarily finite dimensional over $e A e$ $[1$, p. 29, Theorem 3]. As mentioned earlier, Kaplansky [9, Theorem 14] proved that $e A e$ is indeed indiscrete if $A$ is indiscrete and has characteristic zero, and this follows also if $A$ is connected, for then $e A e$ is also connected and hence indiscrete as it is a continuous image of $A$. From Pontrjagin's theorem on connected locally compact division rings, we therefore obtain the following result:

THEOREM 1. If $A$ is a connected locally compact primitive ring having minimal left ideals, then $A$ is isomorphic to the ring of all linear operators on a finite-dimensional vector space over either the field of real numbers, the field of complex numbers, or the division ring of quaternions.

In general, for each $a \in A$ the linear operator $a_{L}$ on $A e$ and the linear operator $a_{R}$ on $e A$ are continuous as multiplication is continuous on $A$. We topologize $A_{L}$ and $A_{R}$ so that the bijections $a \rightarrow a_{L}$ and $a \rightarrow a_{R}$ are homeomorphisms. Both $A_{L}$ and $A_{R}$ are then locally compact rings of continuous linear operators, and since multiplication on $A$ is continuous, $(u, x) \rightarrow u(x)$ is continuous from $A_{L} \times A e$ into $A e$ and also from $A_{R} \times e A$ into $e A$. In particular, for each $x \in A e$ [respectively, $x \in e A$ ], $u \rightarrow u(x)$ is continuous from $A_{L}$ into $A e$ [respectively, from $A_{R}$ into $e A$ ]. 
The vector spaces $A e$ and $e A$ possess an important property defined as follows:

Definition. A topological vector space $E$ over a topological division ring $K$ is straight if for every nonzero vector $x \in E$, the function $\lambda \rightarrow \lambda x$ is a homeomorphism from $K$ onto the one-dimensional subspace of $E$ generated by $x$.

A Hausdorff vector space over an indiscrete topological division ring whose topology is given by an absolute value is straight [1, p. 25, Proposition 2], and any Hausdorff vector space over a (discrete) finite field is clearly straight.

LEMMA 1. If $A$ is a locally compact primitive ring having minimal left ideals and if $e$ is a minimal idempotent of $A$, then the eAe-vector spaces Ae and $e A$ are straight.

Proof. Let $K=e A e$. The function $\lambda \rightarrow e \lambda$ from $K$ onto the subspace $e . K$ of $A e$ is simply the identity mapping of $K$ and hence is a homeomorphism. Let $x$ be a nonzero vector of $A e$. The set of all continuous linear operators on $A e$ is dense since it contains $A_{L}$. In particular, there exist continuous linear operators $u$ and $v$ on $A e$ such that $u(e)=x$ and $v(x)=e$. Thus $e \lambda \rightarrow x \lambda$ is a homeomorphism from $e . K$ onto $x . K$, for it is the restriction of $u$ to $e . K$, and its inverse is the restriction of $v$ to $x . K$. Hence $\lambda \rightarrow x \lambda$ is also a homeomorphism from $K$ onto $x . K$. Similarly, the left $K$-vector space $e A$ is straight.

LEMMA 2. Let E be a discrete topological vector space over a discrete topological division ring $K$, and let $A$ be a set of linear operators on $E$, topologized so that $u \rightarrow u(x)$ is continuous from $A$ into $E$ for each $x \in E$. For every $n \geqq 0$, the set $F_{n}$ of all linear operators in $A$ of rank $\leqq n$ is closed in $A$.

Proof. Let $w$ belong to the closure of $F_{n}$, and let $x_{1}, \ldots, x_{n+1}$ be a sequence of $n+1$ vectors. There is a filter $\mathscr{F}$ on $F_{n}$ converging to $w$, and hence by hypothesis $\mathscr{F}\left(x_{i}\right) \rightarrow w\left(x_{i}\right)$ for $1 \leqq i \leqq n+1$. Since $E$ is discrete, there exists $H_{i} \in \mathscr{F}$ such that $u\left(x_{i}\right)=w\left(x_{i}\right)$ for all $u \in H_{i}$. Let $u \in H_{1} \cap \cdots \cap H_{n+1}$. As $u \in F_{n}$, there exist scalars $\lambda_{1}, \ldots, \lambda_{n+1}$ not all of which are zero such that

$$
\lambda_{1} u\left(x_{1}\right)+\cdots+\lambda_{n+1} u\left(x_{n+1}\right)=0 .
$$

Hence

$$
\sum_{i=1}^{n+1} \lambda_{i} w\left(x_{i}\right)=\sum_{i=1}^{n+1} \lambda_{i} u\left(x_{i}\right)=0
$$

Therefore rank $w \leqq n$.

Lemma 3. Let $E$ be a discrete topological vector space, and let $A$ be a dense ring of linear operators of finite rank on $E$, topologized so that $A$ is a topological ring and $u \rightarrow u(x)$ is continuous from $A$ into $E$ for each $x \in E$. If in addition $A$ is a Baire space and the open additive subgroups of $A$ form a fundamental system of neighborhoods of zero, then $A$ is discrete. 
Proof. If $E$ is finite dimensional, then $A$ is discrete, for if $\left\{c_{1}, \ldots, c_{s}\right\}$ is a basis of $E$, then

$$
\{0\}=\left\{u \in A: u\left(c_{i}\right)=0,1 \leqq i \leqq s\right\}
$$

is a neighborhood of zero since $E$ is discrete. Therefore we shall assume that $E$ is infinite dimensional.

For each $n \geqq 1$ let $F_{n}$ be the set of all linear operators in $A$ of rank $\leqq n$. Then $\bigcup_{n \geqq 1} F_{n}=A$, so by Lemma 2 and our hypotheses there exist $n \geqq 1$, a linear operator $v \in A$, and an open additive subgroup $G$ of $A$ such that $v+G \subseteq F_{n}$. For any $w \in G$,

$$
\operatorname{rank} w \leqq \operatorname{rank}(v+w)+\operatorname{rank}(-v) \leqq n+\operatorname{rank} v
$$

so the ranks of members of $G$ are bounded. Let $m$ be the largest of the ranks of members of $G$, and let $u \in G$ have rank $m$. Let $x_{1}, \ldots, x_{m} \in E$ be such that $\left\{u\left(x_{1}\right), \ldots, u\left(x_{m}\right)\right\}$ is a basis of the range $M$ of $u$. As $E$ is discrete,

$$
V=\left\{v \in G: v\left(x_{i}\right)=0,1 \leqq i \leqq m\right\}
$$

is an open neighborhood of zero in $A$.

We shall show that if $v \in V$, then $v(E) \subseteq M$. If not, let $v \in V$ and $y \in E$ be such that $v(y) \notin M$. Then $u+v \in G$, so rank $(u+v) \leqq m$. But $(u+v)\left(x_{i}\right)=u\left(x_{i}\right)$ if $1 \leqq i \leqq m$, and $(u+v)(y)=u(y)+v(y) \notin M$ since $v(y) \notin M$; hence $u\left(x_{1}\right), \ldots, u\left(x_{m}\right), u(y)+v(y)$ is a linearly independent sequence of $m+1$ vectors belonging to the range of $u+v$, a contradiction.

Since $E$ is infinite dimensional, there exist $y_{1}, \ldots, y_{m} \in E$ such that $u\left(x_{1}\right), \ldots$, $u\left(x_{m}\right), y_{1}, \ldots, y_{m}$ is a linearly independent sequence of $2 m$ vectors. As $A$ is dense, there exists $w \in A$ such that $w\left(u\left(x_{i}\right)\right)=y_{i}$ for $1 \leqq i \leqq m$. As $v \rightarrow w v$ is continuous, there is a neighborhood $U$ of zero in $A$ such that $U \subseteq V$ and $w U \subseteq V$. To show that $U=\{0\}$, let $v \in U$ and let $x \in E$. Then $v(x) \in M$, so there exist scalars $\lambda_{1}, \ldots, \lambda_{m}$ such that

$$
v(x)=\sum_{i=1}^{m} \lambda_{i} u\left(x_{i}\right) .
$$

Consequently,

$$
w v(x)=\sum_{i=1}^{m} \lambda_{i} y_{i}
$$

but $w v(x) \in M$ as $w v \in V$; hence $w v(x)=0$, so $\lambda_{1}=\cdots=\lambda_{m}=0$, whence $v(x)=0$. Thus $U=\{0\}$, so the topology of $A$ is the discrete topology.

LEMMA 4. Let E be a straight locally compact vector space over a discrete topological division ring $K$, and let $A$ be a dense ring of continuous linear operators of finite rank on $E$, topologized so that $A$ is a locally compact ring and $(u, x) \rightarrow u(x)$ is con- 
tinuous from $A \times E$ into $E$. If $E$ is generated by a compact neighborhood $V$ of zero, then $A$ is discrete.

Proof. The ring $A$ is a simple ring having minimal left ideals [6, Theorem 1]; let $e$ be a minimal idempotent of $A$, i.e., a projection on a one-dimensional subspace $M$ of $E$, and let $N$ be the kernel of $e$. As $E$ is straight, $M$ is a discrete subspace and hence is closed, so $V \cap M$ is a compact discrete subset and hence is finite. Therefore there is an open neighborhood $W$ of zero in $E$ such that $W \cap M=\{0\}$. Now $N$ $=e^{-1}(W)$, for if $x \in e^{-1}(W)$, then $e(x) \in W \cap M=\{0\}$, so $x \in N$; but $N=e^{-1}(W)$ is open as $e$ is continuous. As $(u, x) \rightarrow u(x)$ is continuous, the topology of $A$ is stronger than the compact-open topology on $A[4$, p. 46, Corollary 1]. Consequently,

$$
U=\{u \in A: u(V) \subseteq N\}
$$

is a neighborhood of zero in $A$. As $V$ generates $E$,

$$
U=\{u \in A: u(E) \subseteq N\},
$$

so $e U=\{0\}$. Therefore $e A$ is discrete.

As we observed earlier, $A$ is anti-isomorphic as a topological ring to a dense ring $A_{R}$ of linear operators of finite rank on the left $e A e$-vector space $e A$. As $e A$ is discrete, $A$ is not connected; as $A$ is simple, therefore, $A$ is totally disconnected, and thus the open additive subgroups of $A$ form a fundamental system of neighborhoods of zero [2, p. 114, Exercise 18]. Also $A$ is a Baire space as it is locally compact [4, p. 110, Theorem 1]. Thus by Lemma 3 applied to $A_{R}$, we conclude that $A_{R}$ is discrete, whence $A$ is also.

THEOREM 2. A locally compact simple ring A having minimal left ideals is either discrete or isomorphic to the ring of all linear operators on a finite-dimensional vector space over a locally compact division ring.

Proof. Let $e$ be a minimal idempotent of $A$, let $K=e A e$, and let $E$ be the right $K$-vector space $A e$. As observed earlier, the latter conclusion follows if $K$ is indiscrete; therefore we shall assume that $K$ is discrete, and we shall prove that $A$ is discrete. Let $V$ be a compact neighborhood of zero in $E$, which we may assume contains a nonzero vector; then the subspace $F$ generated by $V$ is a nonzero subspace of $E$. Clearly $F$ is a locally compact and hence closed subspace, so the subring

$$
B=\left\{u \in A_{L}: u(F) \subseteq F\right\}
$$

is a closed and hence locally compact subring of $A_{L}$. For each $u \in B$ let $u_{F}$ be the restriction of $u$ to $F$, and let $\rho: u \rightarrow u_{F}$. Then $\rho$ is a homomorphism from $B$ onto a subring $B^{\prime}$ of continuous linear operators of finite rank on $F$, and the kernel of $\rho$ is the ideal

$$
H=\{u \in B: u(F)=\{0\}\}
$$


Now $H$ is clearly closed in $B$, so the topological $\operatorname{ring} B / H$ is Hausdorff and hence locally compact since the canonical epimorphism from $B$ onto $B / H$ is continuous and open. We topologize $B^{\prime}$ so that the algebraic isomorphism from $B / H$ onto $B^{\prime}$ induced by $\rho$ is a homeomorphism; then $\rho$ is a continuous open epimorphism from $B$ onto $B^{\prime}$.

To show that $B^{\prime}$ is a dense ring of linear operators on $F$, let $x_{1}, \ldots, x_{n}$ be a sequence of linearly independent vectors of $F$ and let $y_{1}, \ldots, y_{n} \in F$. Now $A_{L}$ contains a projection $p$ on the subspace generated by $\left\{y_{1}, \ldots, y_{n}\right\}$ [6, Lemma 1], and there exists $u \in A_{L}$ such that $u\left(x_{i}\right)=y_{i}, 1 \leqq i \leqq n$. Then $p u(E) \subseteq F$, so the restriction $v$ of $p u$ to $F$ belongs to $B^{\prime}$ and satisfies $v\left(x_{i}\right)=y_{i}, 1 \leqq i \leqq n$. Moreover, $F$ is straight since $E$ is by Lemma 1 , and $(v, x) \rightarrow v(x)$ is continuous from $B^{\prime} \times F$ into $F$ since $(u, x) \rightarrow u(x)$ is continuous from $A_{L} \times E$ into $E$ and since $\rho$ is an open mapping. Therefore by Lemma $4, B^{\prime}$ is discrete. Consequently, $H$ is open in $B$. But as $V$ generates $F$,

$$
B \supseteq\left\{u \in A_{L}: u(V) \subseteq V\right\},
$$

which is a neighborhood of zero in $A_{L}$ since the topology of $A_{L}$ is stronger than the compact-open topology [4, p. 46, Corollary 1]. Hence $B$ is an open subring of $A_{L}$, so $H$ is an open left ideal of $A_{L}$.

For each $x \in E$, let

$$
H_{x}=\left\{u \in A_{L}: u(x)=0\right\} .
$$

Let $z$ be a nonzero vector of $F$. Then $H_{z} \supseteq H$, and hence $H_{z}$ is an open left ideal of $A_{L}$. Let $x$ be any vector of $E$, and let $g \in A_{L}$ be such that $g(z)=x$. Then there is a neighborhood $W$ of zero in $A_{L}$ such that $W g \subseteq H_{z}$; hence $W \subseteq H_{x}$, so $H_{x}$ is open. We now retopologize $E$ with the discrete topology. Then for each $x \in E, u \rightarrow u(x)$ is continuous from $A_{L}$ into $E$, since $H_{x}$ is open in $A_{L}$. As $K$ is discrete and thus not connected, $A$ and hence $A_{L}$ are not connected; therefore $A_{L}$ is totally disconnected as it is simple, and consequently the open additive subgroups of $A_{L}$ form a fundamental system of neighborhoods of zero [2, p. 114, Exercise 18]. Also $A_{L}$ is a Baire space since it is locally compact $[4$, p. 110 , Theorem 1$]$. Thus by Lemma $3, A_{L}$ is discrete; hence $A$ is discrete.

2. Locally compact vector spaces over discrete division rings. Here we present a few results concerning straight locally compact vector spaces over discrete division rings, and from them we obtain a theorem on locally compact primitive algebras having minimal left ideals.

Lemma 5. If $E$ is a straight Hausdorff topological vector space over a discrete division ring $K$ and if $V$ is a compact subset of $E$, then for every nonzero vector $x \in E,\{\lambda \in K: \lambda x \in V\}$ is finite.

Proof. By hypothesis, $K . x$ is discrete and hence closed, so $V \cap K . x$ is compact and discrete and therefore finite; consequently $\{\lambda \in K: \lambda x \in V\}$ is finite. 
THEOREM 3. Let $E$ be a straight locally compact vector space over a discrete infinite division ring $K$. If $V$ is a compact neighborhood of zero in $E$ and if $\left(\lambda_{n}\right)_{n \geqq 1}$ is any sequence of distinct nonzero scalars, then $\left(\lambda_{1} V \cap \cdots \cap \lambda_{n} V\right)_{n \geqq 1}$ is a fundamental system of neighborhoods of zero in $E$; in particular, $E$ is metrizable.

Proof. For each $n \geqq 1$,

$$
G_{n}=\left\{x \in V: \lambda_{n}^{-1} x \notin V\right\}
$$

is an open subset of $V$, and $\bigcup_{n \geqq 1} G_{n}=V-\{0\}$ by Lemma 5 . Let $U$ be an open neighborhood of zero contained in $V$. Then $V-U$ is compact and is contained in $\bigcup_{n \geqq 1} G_{n}$, so for some $m \geqq 1, V-U \subseteq \bigcup_{k=1}^{m} G_{k}$. Therefore

$$
\begin{aligned}
U \supseteq V-\left(\bigcup_{k=1}^{m} G_{k}\right) & =\bigcap_{k=1}^{m}\left(V-G_{k}\right)=\bigcap_{k=1}^{m}\left\{x \in V: \lambda_{k}^{-1} x \in V\right\} \\
& =V \cap \lambda_{1} V \cap \cdots \cap \lambda_{m} V .
\end{aligned}
$$

Thus $\left(V \cap \lambda_{1} V \cap \cdots \cap \lambda_{n} V\right)_{n \geqq 1}$ is a fundamental system of neighborhoods of zero. Replacing $V$ by $\lambda_{1} V$ and $\lambda_{k} \lambda_{1}^{-1}$, we conclude that $\left(\lambda_{1} V \cap \lambda_{2} V \cap \cdots \cap \lambda_{n} V\right)_{n \geqq 1}$ is a fundamental system of neighborhoods of zero.

THEOREM 4. If $E$ is a straight locally compact vector space over a discrete uncountably infinite division ring $K$, then $E$ is discrete.

Proof. Let $V$ be a compact neighborhood of zero in $E$, let $\left(\lambda_{n}\right)_{n \geqq 1}$ be a sequence of distinct nonzero scalars, and let $V_{m}=\lambda_{1} V \cap \cdots \cap \lambda_{m} V$ for each $m \geqq 1$. For each nonzero scalar $\mu, \mu V$ is a neighborhood of zero, and hence by Theorem 3 there exists $r(\mu) \geqq 1$ such that $V_{r(\mu)} \subseteq \mu V$. Since $K$ is uncountably infinite, there exists $m \geqq 1$ such that $r(\mu)=m$ for infinitely many nonzero scalars $\mu$. If $x$ were a nonzero vector in $V_{m}$, then $x \in \mu V$ and hence $\mu^{-1} x \in V$ for infinitely many nonzero scalars $\mu$, in contradiction to Lemma 5 . Hence $V_{m}=\{0\}$, so $E$ is discrete.

THEOREM 5. If $E$ is a straight locally compact vector space over a discrete division ring $K$ whose characteristic is zero, then $E$ is discrete.

Proof. We assume that $E$ is not discrete.

Case 1. There is a neighborhood of zero in $E$ that contains no nonzero additive subgroup. By a theorem of Gleason [5, Lemma 1.4.2], there is a nonzero continuous homomorphism $\alpha$ from the topological additive group $\boldsymbol{R}$ of real numbers into the additive group $E$. Let $t_{0} \in R$ be such that $\alpha\left(t_{0}\right) \neq 0$, and let $x_{0}=\alpha\left(t_{0}\right)$. Let $\beta$ be the restriction of $\alpha$ to the subgroup of all rational multiples of $t_{0}$. As the characteristic of $K$ is zero, $\beta$ is injective, and its range is discrete as it is a subset of the onedimensional subspace generated by $x_{0}$. Thus $\beta$ is a continuous bijection from an indiscrete group onto a discrete group, which is impossible.

Case 2. Every neighborhood of zero in $E$ contains a nonzero additive subgroup. Let $x_{0}$ be a nonzero member of an additive subgroup $G$ contained in a compact 
neighborhood $V$ of zero. Then $n . x_{0} \in G \subseteq V$ for every integer $n$, so $\lambda x_{0} \in V$ for infinitely many scalars $\lambda$ as the characteristic of $K$ is zero, in contradiction to Lemma 5.

It is easy to construct a straight, indiscrete, compact vector space over a discrete finite field: we need only consider the cartesian product of infinitely many copies of the field. By Theorems 4 and 5, there is no straight, indiscrete, locally compact vector space over a discrete division ring $K$ if either $K$ is uncountably infinite or $K$ has characteristic zero. We next construct an example of a straight, indiscrete, locally compact vector space over a discrete, countable division ring of prime characteristic.

ExAmple 1. Let $P$ be a prime field of prime characteristic, and let $K$ be a countably infinite algebraic extension of $P$, e.g., let $K$ be an algebraic closure of $P$. Let $\lambda_{1}, \lambda_{2}, \ldots$ be an enumeration of the nonzero elements of $K$, let $K_{0}=P$, and for each $n \geqq 1$ let $K_{n}=P\left[\lambda_{1}, \ldots, \lambda_{n}\right]$, the subfield generated by $\lambda_{1}, \ldots, \lambda_{n}$. As $K$ is an algebraic extension of $P,\left(K_{n}\right)_{n \geqq 0}$ is an increasing sequence of finite subfields of $K$ whose union is $K$. Let $E=K^{N}$, the $K$-vector space of all sequences $\left(\alpha_{n}\right)_{n \geq 0}$ where $\alpha_{n} \in K$ for all $n \geqq 0$. Let $V=\prod_{n \geqq 0} K_{n}$, and for each $m \geqq 1$ let $V_{m}=\lambda_{1} V \cap \ldots$ $\cap \lambda_{m} V$. We shall prove that $\left(V_{m}\right)_{m \geqq 1}$ is a fundamental system of neighborhoods of zero for a topology on $E$ making $E$ an indiscrete, straight locally compact vector space over the discrete field $K$.

Since each $V_{m}$ is an additive subgroup, addition is continuous on $E$. As $K$ is discrete, to show that scalar multiplication is continuous it suffices to show that $x \rightarrow \lambda x$ is continuous at zero for each nonzero scalar $\lambda$. But if $m \geqq 1$, then $\lambda V_{r} \subseteq V_{m}$ where $r$ is such that $\lambda^{-1} \lambda_{1}, \ldots, \lambda^{-1} \lambda_{m}$ are among $\lambda_{1}, \ldots, \lambda_{r}$. Thus $E$ is a topological $K$-vector space.

To show that our topology is stronger than the cartesian product topology on $E=K^{N}$, let $W=\prod_{n \geqq 0} L_{n}$ where $L_{n}=\{0\}$ for $n<m$ and $L_{n}=K$ for $n \geqq m$. Let $\beta \in K$ be such that $\beta \notin K_{m}$; to show that $V \cap \beta V \subseteq W$, let $\left(\alpha_{n}\right)_{n \geqq 0} \in V \cap \beta V$. If $n<m$, then $\alpha_{n} \in K_{n}$ and $\beta^{-1} \alpha_{n} \in K_{n}$, so if $\alpha_{n} \neq 0$, then $\beta^{-1}$ and hence also $\beta$ would belong to $K_{n} \subseteq K_{m}$, a contradiction. Hence $\alpha_{n}=0$ if $n<m$, so $\left(\alpha_{n}\right)_{n \geqq 0} \in W$, and thus $V \cap \beta V \subseteq W$. In particular, our topology is a Hausdorff topology.

Next we shall show that the topology induced on $V$ is identical with the cartesian product topology of $V=\prod_{n \geqq 0} K_{n}$. By the preceding, it suffices to show that for each $m \geqq 1$ we have $H=\prod_{n \geqq 0} H_{n} \subseteq V_{m}$ where $H_{n}=\{0\}$ if $n<m$ and $H_{n}=K_{n}$ if $n \geqq m$. Let $\left(\alpha_{n}\right) \in H$, and let $n \geqq m$. Then $\alpha_{n} \in K_{n}$ and $\lambda_{i}^{-1} \in K_{m} \subseteq K_{n}$ if $1 \leqq i \leqq m$, so $\lambda_{i}^{-1} \alpha_{n} \in K_{n}$ if $1 \leqq i \leqq m$, and thus $\alpha_{n} \in \lambda_{1} K_{n} \cap \cdots \cap \lambda_{m} K_{n}$. Therefore

$$
\left(\alpha_{n}\right) \in \prod_{n \geqq 0}\left(\lambda_{1} K_{n} \cap \cdots \cap \lambda_{m} K_{n}\right)=\lambda_{1} V \cap \cdots \cap \lambda_{m} V=V_{m} .
$$

In particular, our topology is indiscrete and $V$ is compact for it; thus $E$ is an indiscerte locally compact $K$-vector space.

It remains for us to show that $E$ is straight, i.e., that every one-dimensional 
subspace of $E$ is discrete. Let $z=\left(\alpha_{n}\right)_{n \geqq 0}$ be a nonzero vector, let $\alpha_{s} \neq 0$, and let $L=\{\lambda \in K: \lambda z \in V\}$. Then $L \alpha_{s} \subseteq K_{s}$, so $L$ is finite since $\alpha_{s} \neq 0$ and $K_{s}$ is finite. Therefore $K . z \cap V$ is finite, so $K . z$ is discrete.

To apply these results to locally compact primitive algebras having minimal left ideals, we first note that if $A$ is a primitive algebra, then a minimal left ideal of the algebra $A$ is also a minimal left ideal of the ring $A$ since the right annihilator of $A$ is the zero ideal. Therefore a primitive algebra having minimal left (algebra) ideals is also a primitive ring having minimal left ideals.

THEOREM 6. Let A be a locally compact primitive algebra having minimal left ideals over a topological field $K$. If any one of the following three conditions holds, then either $A$ is discrete or $A$ contains an identity element and is finite-dimensional over its center.

$1^{\circ} K$ is indiscrete.

$2^{\circ} K$ has characteristic zero.

$3^{\circ} K$ is uncountably infinite.

Proof. Let $e$ be a minimal idempotent of $A$, and let $D=e A e$.

Case $1 . D$ is indiscrete. Then $D$ is an indiscrete locally compact division ring and hence is finite dimensional over its center [8, Theorem 8], and as we observed earlier $A$ is isomorphic to the ring of all linear operators on a finite-dimensional $D$-vector space. Hence $A$ has an identity element and the center of $D$ may be identified with the center of $A$, so $A$ is finite dimensional over its center.

Case 2. $D$ is discrete. As $\lambda \rightarrow \lambda e$ is a continuous injection from $K$ into $D, K$ is also discrete and hence by our hypothesis either has characteristic zero or is uncountably infinite. To show that the $K$-vector space $A$ is straight, let $a$ be a nonzero element of $A$, and let $x \in A e$ be such that $a_{L}(x) \neq 0$. As we saw earlier, $v \rightarrow v(x)$ is continuous from $A_{L}$ into $A e$, and its restriction to $(K . a)_{L}$ is an injection from $(K . a)_{L}$ into $a_{L}(x) . D$, since

$$
(\lambda a)_{L}(x)=(\lambda a) x=(\lambda a)(x e)=(a x)(\lambda e) \in a_{L}(x) \cdot D
$$

as $x=x e$. By Lemma $1, a_{L}(x) . D$ is discrete, so $K . a$ is also discrete. Therefore $A$ is straight, so by Theorems 4 and $5, A$ is discrete.

COROLlARY. If $A$ is a locally compact central primitive algebra having minimal left ideals over a topological field $K$ and if $K$ either is indiscrete, has characteristic zero, or is uncountably infinite, then $A$ is finite dimensional over $K$.

Proof. If $A$ is indiscrete, then by Theorem $6 A$ has an identity element 1 , and hence $\lambda \rightarrow \lambda .1$ is a bijection from $K$ onto the center of $A$ as $A$ is central; therefore $A$ is finite dimensional over $K$ by Theorem 6.

Kaplansky [9, p. 458] constructed for any finite field $K$ an indiscrete locally compact primitive $K$-algebra that has an identity element and minimal left ideals 
but is infinite dimensional over its center. We construct another example of such an algebra where $K$ is countably infinite and has prime characteristic.

EXAMPLE 2. We continue with the notation of Example 1. Let $F$ be the subspace of $E$ generated by $V$. Then $F$ is locally compact, metrizable, and

$$
F=\bigcup_{n \geqq 1}\left(\lambda_{1} V+\cdots+\lambda_{n} V\right) \text {, }
$$

the union of a countable sequence of compact open subgroups; hence $F$ is also separable [3, p. 43, Corollary]. Consequently $\mathscr{C}(F)$, the $K$-algebra of all continuous functions from $F$ into $F$, equipped with the compact-open topology, is a separable, metrizable, topological $K$-algebra [4, p. 47, Proposition 9; p. 34, Corollary; p. 41, Corollary]. We shall show that the subalgebra $A$ of all continuous linear operators on $F$ is indiscrete and locally compact. Clearly $A$ is a closed subalgebra of $\mathscr{C}(F)$. Let

$$
U=\{u \in A: u(V) \subseteq V\} .
$$

Then $U$ is a neighborhood of zero in $A$, and $U$ is clearly closed in $A$ and hence in $\mathscr{C}(F)$. Also, $U$ is equicontinuous, for $u\left(V_{m}\right) \subseteq V_{m}$ for all $u \in U$ and all $m \geqq 1$. To show that $U$ is compact, therefore, it suffices by Ascoli's Theorem [4, p. 32, Corollary 3] to show that $U(x)$ is relatively compact for each $x \in F$. But if $x \in F$, then $x \in \lambda_{1} V+\cdots+\lambda_{m} V$ for some $m \geqq 1$, whence $u(x) \in \lambda_{1} V+\cdots+\lambda_{m} V$ for all $u \in U$; thus $U(x) \subseteq \lambda_{1} V+\cdots+\lambda_{m} V$, a compact set. Hence $A$ is a locally compact, separable, metrizable $K$-algebra.

For each $m \geqq 0$ let $e_{m}$ be the projection defined by

$$
e_{m}\left(\left(\alpha_{n}\right)\right)=\left(\delta_{n m} \alpha_{n}\right)
$$

for all $\left(\alpha_{n}\right) \in F$, where $\delta_{n m}$ is the Kronecker notation. Then $e_{m}^{-1}(V) \supseteq V$, so $e_{m}^{-1}\left(V_{r}\right) \supseteq V_{r}$ for all $r \geqq 1$, and hence $e_{m} \in A$. Thus $A$ contains nonzero linear operators of finite rank. To show that $A$ is dense, it suffices to show that $A$ is 2-fold transitive. Let $\left\{\left(\alpha_{n}\right),\left(\beta_{n}\right)\right\}$ be a linearly independent set of two vectors of $F$. If $\alpha_{m}=0$ and $\beta_{m} \neq 0$ for some $m \geqq 0$, then $e_{m}\left(\left(\alpha_{n}\right)\right)=0$ and $e_{m}\left(\left(\beta_{n}\right)\right) \neq 0$; otherwise $\alpha_{m}=0$ implies that $\beta_{m}=0$ for all $m \geqq 0$, so there exist $r, s$ such that $\alpha_{r} \neq 0, \alpha_{s} \neq 0$, and $\beta_{r} / \alpha_{r} \neq \beta_{s} / \alpha_{s}$, whence $u=\alpha_{r}^{-1} e_{r}-\alpha_{s}^{-1} e_{s}$ satisfies $u\left(\left(\alpha_{n}\right)\right)=0, u\left(\left(\beta_{n}\right)\right) \neq 0$. Since $A$ contains the identity linear operator $1, \lambda \rightarrow \lambda .1$ is therefore an isomorphism from $K$ onto the center of $A$. Therefore $A$ is an infinite-dimensional central primitive $K$-algebra having minimal left ideals and an identity element.

It remains for us to show that $A$ is indiscrete; we shall show that $e_{m} \rightarrow 0$. Every compact subset of $F$ is contained in $\lambda_{1} V+\cdots+\lambda_{n} V$ for some $n \geqq 1$. If $m \geqq n$, then $\lambda_{1}, \ldots, \lambda_{n} \in K_{m}$, and hence $e_{m}\left(\lambda_{1} V+\cdots+\lambda_{n} V\right) \subseteq \lambda_{1} V \cap \cdots \cap \lambda_{n} V$. Therefore $e_{m} \rightarrow 0$.

\section{REFERENCES}

1. N. Bourbaki, Espaces vectoriels topologiques, Chapters 1-2, Hermann, Paris, 1953.

2. —, Topologie générale, Chapters 3-4, 3rd ed., Hermann, Paris, 1960. 
3. - Topologie générale, Chapter 9, 2nd ed., Hermann, Paris, 1958.

4. —, Topologie générale, Chapter 10, 2nd ed., Hermann, Paris, 1961.

5. Andrew M. Gleason, Groups without small subgroups, Ann. of Math. 56 (1952), 193-212.

6. Nathan Jacobson, Structure theory of simple rings without finiteness assumptions, Trans. Amer. Math. Soc. 57 (1945), 228-245.

7. - Structure of rings, Amer. Math. Soc. Colloq. Publ. Vol. 37, Amer. Math. Soc., Providence, R. I., 1956.

8. Irving Kaplansky, Topological methods in valuation theory, Duke Math. J. 14 (1947), 527-541.

9. - Locally compact rings, Amer. J. Math. 70 (1948), 447-459.

10. Yosikazu Otobe, On locally compact fields, Japan. J. Math. 19 (1945), 189-202.

DUKe UNIVERSITY,

Durham, North Carolina 